3. - Higher-dimensional hereditarily indecomposable continua, Trans. Amer. Math. Soc. vol. 71 (1951) pp. 267-273.

4. - Snake-like continua, Duke Math. J. vol. 18 (1951) pp. 653-663.

5. J. L. Kelley, The hyperspaces of a continuum, Trans. Amer. Math. Soc. vol. 52 (1942) pp. 22-36.

6. B. Knaster, Un continu dont tout sous-continu est indécomposable, Fund. Math. vol. 3 (1922) pp. 247-286.

7. E. E. Moise, An indecomposable plane continuum which is homeomorphic to each of its nondegenerate subcontinua, Trans. Amer. Math. Soc. vol. 63 (1948) pp. 581-594.

8. R. L. Moore, Foundations of point set theory, Amer. Math. Soc. Colloquium Publications, vol. 13, New York, 1932.

9. G. T. Whyburn, Analytic topology, Amer. Math. Soc. Colloquium Publications, vol. 28, New York, 1942.

UNIVERSITY OF WISCONSIN

\title{
UNE PROPRIETE TOPOLOGIQUE DES DOMAINES DE RUNGE
}

\section{JEAN-PIERRE SERRE}

Nous dirons qu'un domaine $X$ de l'espace numérique complexe $\boldsymbol{C}^{n}$ est un domaine de Runge si:

1. $X$ est un domaine d'holomorphie.

2. Toute fonction holomorphe sur $X$ est limite uniforme sur tout compact de polynômes.

On sait que, si $n=1, X$ est simplement connexe. Nous allons généraliser ce résultat:

ThÉORÈme. Le $n^{\text {eme }}$ nombre de Betti d'un domaine de Runge de $\mathrm{C}^{n}$ est nul.

Démonstration. Soit $C^{n}(X)$ l'espace vectoriel des formes différentielles $\omega=f\left(z_{1}, \cdots, z_{n}\right) d z_{1} \wedge \cdots \wedge d z_{n}$, où $f$ est holomorphe sur $X$. Une telle forme est toujours fermée, i.e. $d \omega=0$. Soit $B^{n}(X)$ le sous-espace de $C^{n}(X)$ formé des éléments $\omega$ qui sont de la forme $d \alpha$, où $\alpha$ est une forme différentielle holomorphe de degré $n-1$. D'après le Théorème 1 de [2], qui s'applique à cause de l'hypothèse 1 , l'espace quotient $C^{n}(X) / B^{n}(X)$ est isomorphe à $H^{n}(X, C)$, et tout revient donc à montrer que $B^{n}(X)=C^{n}(X)$.

Munissons $C^{n}(X)$ de la topologie de la convergence compacte. Alors:

(a) $B^{n}(X)$ est fermé dans $C^{n}(X)$.

Received by the editors April 24, 1954. 
En effet, d'après [2, no. 3], pour qu'un élément $\omega$ de $C^{n}(X)$ appartienne à $B^{n}(X)$, il faut et il suffit que ses périodes $\int_{c} \omega$ sur les cycles différentiables $c$ de $X$ soient nulles. Notre assertion résulte alors de la continuité de l'application $\omega \rightarrow \int_{c} \omega$.

(b) $B^{n}(X)$ est dense dans $C^{n}(X)$.

En effet, l'hypothèse 2 signifie que les formes $\omega=P \cdot d z_{1} \wedge \cdots \wedge d z_{n}$, où $P$ est un polynôme en $z_{1}, \cdots, z_{n}$, sont denses dans $C^{n}(X)$. Or une telle forme appartient à $B^{n}(X)$, car on peut écrire $P=\partial Q / \partial z_{1}$, où $Q$ est un polynôme, et l'on a

$$
P \cdot d z_{1} \wedge \cdots \wedge d z_{n}=d\left(Q \cdot d z_{2} \wedge \cdots \wedge d z_{n}\right) .
$$

Les propriétés (a) et (b) entraînent $B^{n}(X)=C^{n}(X)$, cqfd.

Remarques. (1) Il existe des domaines de Runge de $C^{n}, n>1$, qui ne sont pas simplement connexes; on en trouvera un exemple simple dans [1, p. 209].

(2) Le théorème démontré ci-dessus équivaut à dire que $H_{n}(X)$ est un groupe de torsion. En fait, je ne connais aucun exemple dans lequel ce groupe ne soit pas nul.

\section{BiBLIOGRAPHIE}

1. H. Behnke und K. Stein, Konvergente Folgen von Regularitätsbereichen und die Meromorphiekonvexität, Math. Ann. vol. 116 (1939) pp. 204-216.

2. J-P. Serre, Quelques problèmes globaux relatifs aux variétés de Stein, Colloque de Bruxelles, 1953, pp. 57-68.

PARIS 